\title{
Top quark pair property measurements using the ATLAS detector at the LHC
}

\author{
Mohammad J. Kareem*i \\ II. Physikalisches Institut, Georg-August-Universität Göttingen, \\ Friedrich-Hund-Platz 1, D-37077 Göttingen, Germany \\ E-mail: mohammad.kareemecern.ch
}

\begin{abstract}
Precise measurements of the properties of the top quark test the Standard Model (SM) and can be used to constrain new physics models. The top quark pair charge asymmetry is an asymmetry predicted to occur beyond leading-order QCD in the SM, and may be significantly enhanced by the presence of new physics. The $t \bar{t}$ production charge asymmetry is measured inclusively and differentially using the $8 \mathrm{TeV}$ ATLAS dataset in the lepton+jets final state, including a dedicated measurement for highly boosted top quarks. The results are in agreement with the SM and compared to new physics models. The top quark is predicted in the SM to decay almost exclusively to a $W$ boson and $b$ quark. Searches for non-SM top quark decays using the $8 \mathrm{TeV}$ ATLAS dataset, including $t \rightarrow q H$ and $t \rightarrow q Z$ are presented. In addition, the measurement of the spin correlations in top quark pair production using the polar angles distribution is discussed.
\end{abstract}

XXIV International Workshop on Deep-Inelastic Scattering and Related Subjects

11-15 April, 2016

DESY Hamburg, Germany

\footnotetext{
*Speaker.

On behalf of the ATLAS Collaboration
} 


\section{Introduction}

Discovered in 1995 at the Tevatron $p \bar{p}$ collider by the CDF and D0 collaborations [1,2], the top quark is known as the heaviest particle in the Standard Model (SM) of particle physics. In the Large Hadron Collider (LHC) [3], top quarks are produced in pairs through the strong interaction and individually through electroweak processes via proton-proton collisions. With a mass around 173 $\mathrm{GeV}$ close to the electroweak symmetry breaking scale, measurements of the top quark properties can provide an important tool in terms of tests of the Standard Model. The top quark has an extremely short life time that leads to a decay before hadronization, providing a unique opportunity to study the bare quark properties. Due to the high production rate of the top quark at the LHC, top quark properties measurements are of great importance.

In this context, using data taken at centre-of-mass energies of $\sqrt{s}=7 \mathrm{TeV}$ and $8 \mathrm{TeV}$, a variety of analyses are carried out by the ATLAS collaboration [4] where some of the most recently obtained results are discussed here.

\section{Charge asymmetry}

The production rate difference in positive and negative absolute rapidity difference between top quarks and top antiquarks known as charge asymmetry $\left(A_{C}\right)$, defined as

$$
A_{C}=\frac{N(\Delta|y|>0)-N(\Delta|y|<0)}{N(\Delta|y|>0)+N(\Delta|y|<0)}
$$

is one of the interesting features of $t \bar{t}$ production in $p \bar{p}$ collisions. The Standard Model at next-toleading order (NLO) in Quantum Chromodynamics (QCD) predicts a charge asymmetry at the level of $A_{C} \sim 1 \%$. On the other hand, several processes beyond the Standard Model (BSM) can alter $A_{C}$, either with anomalous vector or axial-vector couplings (e.g. axi-gluons) or via interference with SM processes, predicting different asymmetries.

One of the latest charge asymmetry measurements at ATLAS uses the full $\sqrt{s}=8 \mathrm{TeV}$ data set corresponding to an integrated luminosity of $20.3 \mathrm{fb}^{-1}$ in the single-lepton final state (where one top quark decays to a leptonically decaying $W$ boson and the other top quark decays to a hadronically decaying $W$ boson). The result was obtained inclusively and differentially as a function of invariant mass, transverse momentum and longitudinal boost $\left(\beta_{Z}\right)$ of the $t \bar{t}$ system, requiring at least four jets, one high $p_{T}$ lepton and missing transverse energy. The events are reconstructed via a kinematic likelihood fit method [5] and a Bayesian unfolding procedure is applied to account for distortions due to the acceptance and detector effects, leading to parton-level $A_{C}$ measurements. The inclusive measurement yields a value of $A_{C}=0.009 \pm 0.005$ (stat. + syst.) [6].

In addition, ATLAS performed the charge asymmetry measurement in the boosted topology [7], by reconstructing the hadronic hemisphere of the $t \bar{t}$ event as one large-radius jet with radius parameter $R=1.0$ and $p_{T}>300 \mathrm{GeV}$ inclusively and differentially. The boosted topology provides an accurate $A_{C}$ measurement as a function of the $t \bar{t}$ invariant mass $\left(m_{t \bar{t}}\right)$ in the $\mathrm{TeV}$ range by a more precise reconstruction of $m_{t \bar{t}}$ for events with highly boosted top quarks. The inclusive measurement for $m_{t \bar{t}}>0.75 \mathrm{TeV}$ and $|\Delta| y||<2$ yields $A_{C}=(4.2 \pm 3.2) \%$, that is within one standard deviation of the SM expectation. Furthermore, the differential measurement as function of the invariant mass of the $t \bar{t}$ system disfavours t-channel $\mathrm{W}^{\prime}$ boson model [8] in the highest $m_{t \bar{t}}$ bin. 
Figure 1 shows the differential $A_{C}$ measurement in resolved and boosted topologies as a function of $m_{t \bar{t}}$. These measurements provide a constraint on extensions of the SM. The results of both measurements agree with the SM prediction.
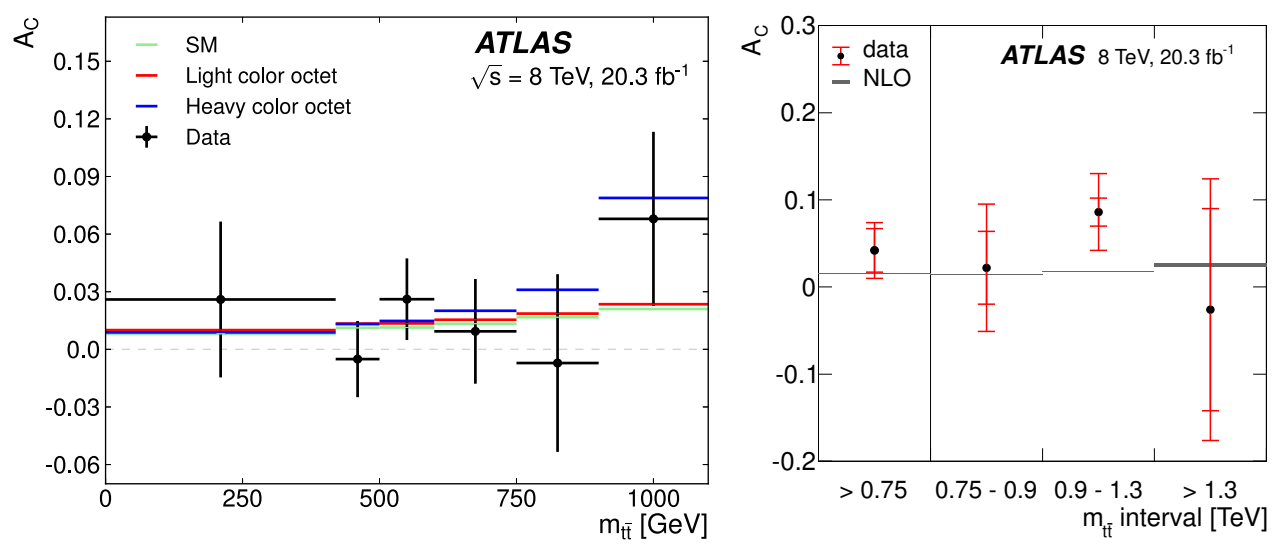

Figure 1: The $A_{C}$ measurement in resolved topology [6] (left), compared with predictions for SM and for right-handed colour octets with masses below the $t \bar{t}$ threshold and beyond the kinematic reach of current LHC searches and boosted topology [7] (right) as a function of $m_{t \bar{t}}$ compared with the SM prediction of the NLO calculation.

\section{Rare decays of the top quark}

Within the Standard Model, flavour changing neutral currents (FCNC) are forbidden at tree level and are heavily suppressed via the GIM mechanism [9]. In contrast, many BSM models predict significant enhancements at the level of the experimental accessibility, making this top quark property measurements an area of high interest. Within this context, ATLAS performed various searches for FCNC processes such as recent searches for $\mathscr{B}(t \rightarrow q H)$ [10] and $\mathscr{B}(t \rightarrow q Z)$ [11], using $t \bar{t}$ events produced in the full $\sqrt{s}=8 \mathrm{TeV}$ data set corresponding to an integrated luminosity of $20.3 \mathrm{fb}^{-1}$, with one top quark decaying through the FCNC mode and the other through the SM dominant mode $(t \rightarrow b W)$. Only the decays of the Higgs boson to $b \bar{b}$ and the $\mathrm{Z}$ boson to charged leptons and leptonic $W$ boson decays are considered, respectively. The final state topology of the top decay through the $t q H$ process is characterised by an isolated high transverse momentum lepton and at least four jets. The final state characteristics of top quark decays through the $t q Z$ process is given by three isolated charged leptons, at least two jets, and missing transverse momentum from the undetected neutrino.

For the $t q H$ process, results from other ATLAS searches with $H \rightarrow \gamma \gamma$ and $H \rightarrow W^{+} W^{-}, \tau^{+} \tau^{-}$ have been combined with $H \rightarrow b \bar{b}$ to obtain the most restrictive direct bounds on $t q H$ interactions measured so far. Figure 2 summarises the best fit for the individual searches as well as their combination.

No evidence for signal events above the background expectation is found, but the established limits for these FCNC processes are in agreement with the expected theoretical limits. 

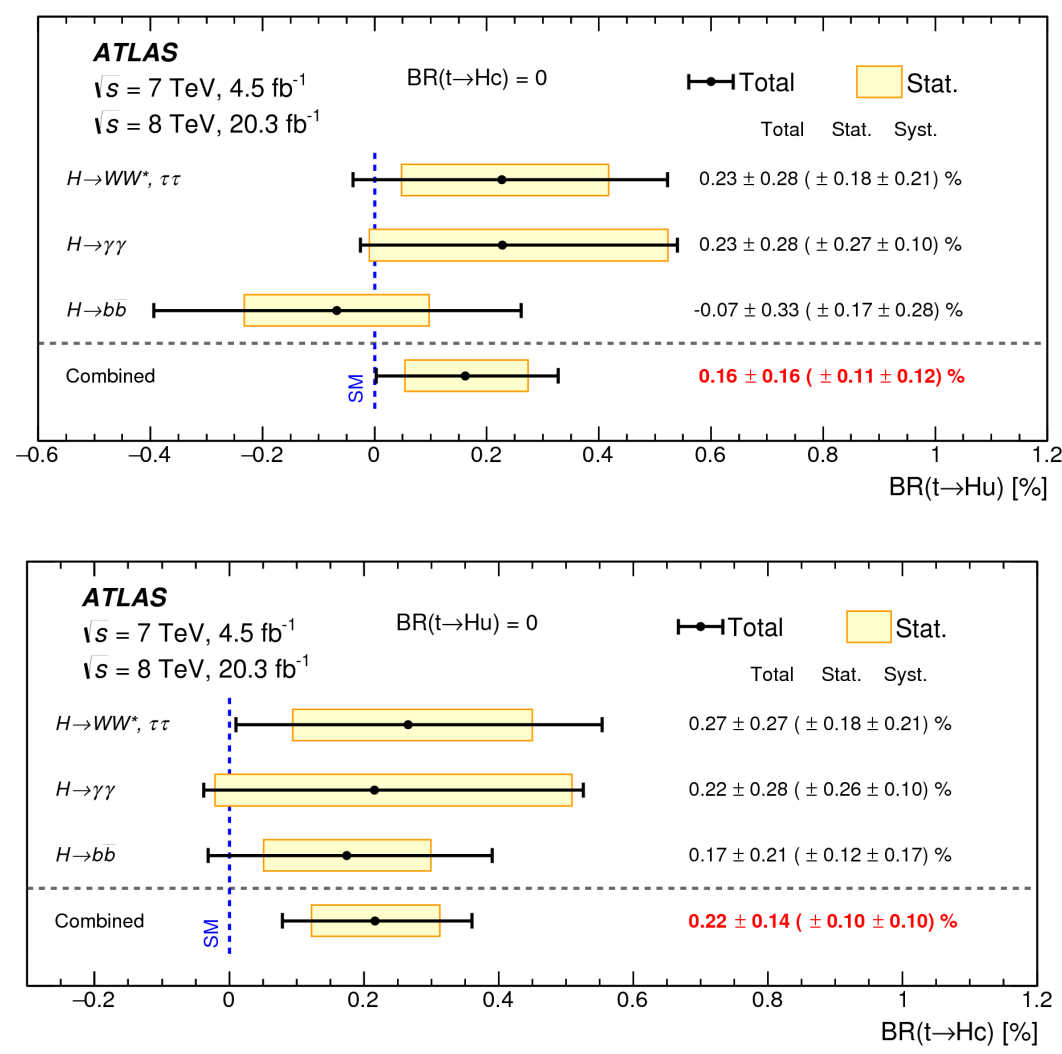

Figure 2: The best-fit for the individual searches as well as their combination for (top) $\mathscr{B}(t \rightarrow H u)$, assuming that $\mathscr{B}(t \rightarrow H c)=0$ and (bottom) $\mathscr{B}(t \rightarrow H c)$, assuming that $\mathscr{B}(t \rightarrow H u)=0$ [10].

\section{Spin correlation}

Because of the extreme short lifetime of the top quark, it decays before hadronization and that implies that the spin information of the top quark can be accessed from the angular momentum distributions of its decay products. The degree of correlation between the spin of the top quark and the top anti-quark is sensitive to the production mechanism. However, many scenarios of physics beyond the Standard Model predict different spin correlations, e.g. models including axi-gluons, $W^{\prime}$ bosons, extra right handed top-quark coupling, etc.

A recent measurement of the correlations between the polar angles of leptons from the decay of top quarks in $t \bar{t}$ events in the helicity basis carried out at the ATLAS experiment [12]. The data set corresponds to an integrated luminosity of $4.6 \mathrm{fb}^{-1}$ at a centre-of-mass energy of $\sqrt{s}=7$ $\mathrm{TeV}$ with candidate events selected in the dilepton topology (where both top quarks in the $t \bar{t}$ event decay to a leptonically decaying $W$ boson) with large missing transverse momentum and at least two jets. The angles $\theta_{1}$ and $\theta_{2}$ between the charged leptons and the direction of motion of the parent quarks in the $t \bar{t}$ system rest frame are sensitive to the spin information, and the distribution of $\cos \theta_{1} \cdot \cos \theta_{2}$ is sensitive to the spin correlation between the top quark and the top anti-quark. The events are reconstructed via the so called topology reconstruction method and the result is 
unfolded to the parton level using a fully Bayesian unfolding algorithm. The unfolded distribution is in good agreement with the prediction from MC@NLO [13] as displayed in Fig. 3.

In terms of $A_{\text {helicity }}=\left(N_{\text {like }}-N_{\text {unlike }}\right) /\left(N_{\text {like }}+N_{\text {unlike }}\right)$, where $N_{\text {like }}\left(N_{\text {unlike }}\right)$ is the number of events where the spins of the top quark and top anti-quark are (anti-)parallel with respect to the helicity basis, the result yields a value of $A_{\text {helicity }}=0.315 \pm 0.061$ (stat. $) \pm 0.049$ (syst.), and is in a good agreement with the NLO QCD prediction of $A_{\text {helicity }}=0.31$ [14].

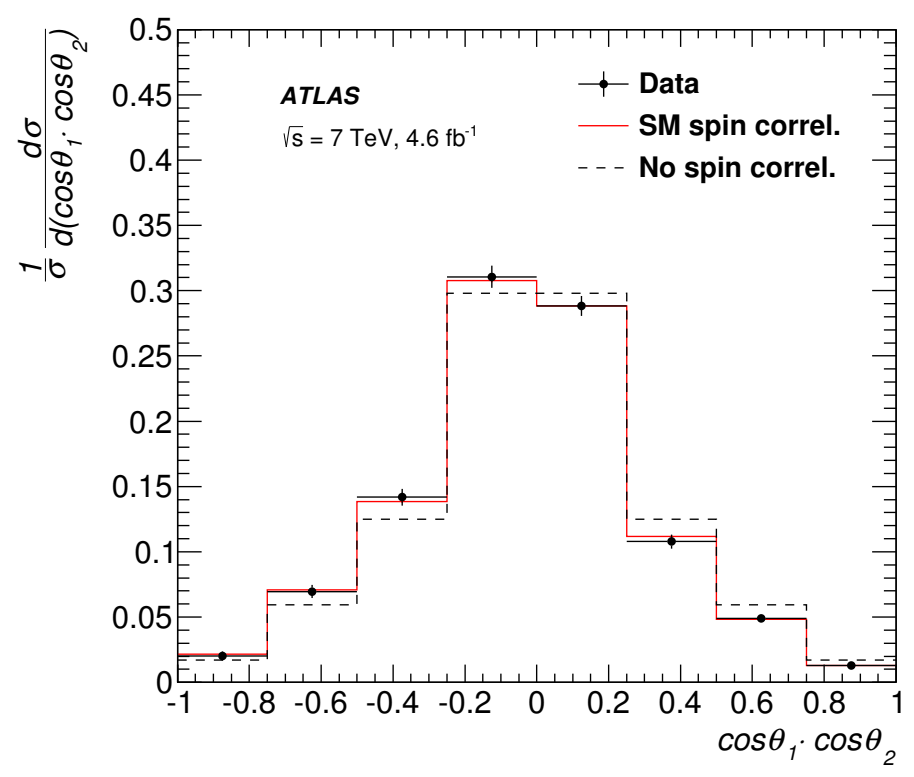

Figure 3: The unfolded data distribution of $\cos \theta_{1} \cdot \cos \theta_{2}$. The predictions from the SM and the MC@NLO sample without spin correlation are overlaid for comparison. A symmetric distribution around zero would indicate no spin correlation [12].

\section{Conclusion}

Measuring top quark properties with high precision is a very good probe for the Standard Model and could potentially open a window to beyond Standard Model physics. A variety of recent measurements of top quark properties at ATLAS are discussed. Inclusive and differential charge asymmetry measurements in resolved and boosted topologies in the single lepton final state at a centre-of-mass energy of $\sqrt{s}=8 \mathrm{TeV}$ are presented. The measurement with boosted topology extended the reach of previous ATLAS and CMS [15] measurements to beyond $1 \mathrm{TeV}$ and disfavours a t-channel $\mathrm{W}^{\prime}$ boson model in the highest $m_{t \bar{t}}$ bin. However, no significant deviation from the SM expectation is observed. Having the data statistics as the limiting factor in these measurements, analysing the LHC Run II data with higher centre-of-mass energy of collision and luminosity will be of great interest. Furthermore, several searches for the observation of the flavour changing neutral current processes carried out with no significant evidence. An increased amount of data and improved measurement techniques will soon improve the current limits. The measurement of the 
correlations between the polar angles of leptons from the decay of the pair-produced top quark and top antiquark in the helicity basis in dilepton final state at a centre-of-mass energy of $\sqrt{s}=7 \mathrm{TeV}$ confirms the spin correlation in top quark decay and is in good agreement with the predictions of the Standard Model.

\section{References}

[1] CDF collaboration, F. Abe et al., Observation of top quark production in $\bar{p} p$ collisions, Phys. Rev. Lett. 74 (1995) 2626.

[2] D0 collaboration, S. Abachi et al., Observation of top quark, Phys. Rev. Lett. 74 (1995) 2632.

[3] L. Evans and P. Bryant, LHC Machine, JINST 3 (2008) S08001.

[4] ATLAS Collaboration, The ATLAS Experiment at the CERN Large Hadron Collider, JINST 3 (2008) S08003.

[5] J. Erdmann et al., A likelihood-based reconstruction algorithm for top-quark pairs and the KLFitter framework, Nucl. Instrum. Meth. A748 (2014) 18-25.

[6] ATLAS collaboration, Measurement of the charge asymmetry in top-quark pair production in the lepton-plus-jets final state in pp collision data at $\sqrt{s}=8 \mathrm{TeV}$ with the ATLAS detector, Eur. Phys. J. C76 (2016) 87.

[7] ATLAS collaboration, Measurement of the charge asymmetry in highly boosted top-quark pair production in $\sqrt{s}=8 \mathrm{TeV}$ pp collision data collected by the ATLAS experiment, Phys. Lett. $\mathbf{B} 756$ (2016) 52-71.

[8] J. A. Aguilar-Saavedra and M. Perez-Victoria, Asymmetries in t $\bar{t}$ production: LHC versus Tevatron, Phys. Rev. D84 (2011) 115013.

[9] S. L. Glashow, J. Iliopoulos and L. Maiani, Weak interactions with lepton-hadron symmetry, Phys. Rev. D 2 (1970) 1285.

[10] ATLAS collaboration, Search for flavour-changing neutral current top quark decays $t \rightarrow H q$ in $p p$ collisions at $\sqrt{s}=8 \mathrm{TeV}$ with the ATLAS detector, JHEP 12 (2015) 061.

[11] ATLAS collaboration, Search for flavour-changing neutral current top-quark decays to $q Z$ in $p p$ collision data collected with the ATLAS detector at $\sqrt{s}=8 \mathrm{TeV}$, Eur. Phys. J. C76 (2016) 12.

[12] ATLAS collaboration, Measurement of the correlations between the polar angles of leptons from top quark decays in the helicity basis at $\sqrt{s}=7 T e V$ using the ATLAS detector, Phys. Rev. D93 (2016) 012002.

[13] S. Frixione, P. Nason and B. R. Webber, Matching NLO QCD and parton showers in heavy flavor production, JHEP 08 (2003) 007.

[14] W. Bernreuther, A. Brandenburg, Z. G. Si and P. Uwer, Top quark spin correlations at hadron colliders: Predictions at next-to-leading order QCD, Phys. Rev. Lett. 87 (2001) 242002.

[15] CMS collaboration, V. Khachatryan et al., Measurement of the charge asymmetry in top quark pair production in pp collisions at $\sqrt{(s)}=8 \mathrm{TeV}$ using a template method, Phys. Rev. D93 (2016) 034014. 